\title{
Variabilidad espacial de las propiedades físicas de un suelo Fluventic Ustropepts en la cuenca baja del río Las Ceibas - Huila
}

\author{
Spatial variability of the physical properties of \\ Fluventic Ustropepts soil in the lower basin of the \\ Las Ceibas River - Huila
}

\author{
Rocío del Pilar Vargas S. ${ }^{[1]}$, Fernando Serrato C. ${ }^{[2]}$ y Armando Torrente T. ${ }^{[3]}$
}

\begin{abstract}
Resumen
Se estudió la variabilidad espacial de algunas propiedades físicas del suelo asociadas a cultivos de caña, pasto y moringa en un "Fluventic Ustropepts" de la Cuenca baja del Rio Las Ceibas. Se hizo muestreo con grilla de 50 m x 50 m en 26 puntos para los análisis físicos, 8 pruebas de infiltración en muestreo aleatorio simple y 3 muestras integradas para análisis químicos en un área de 6.5 ha. El estudio consta de 5 etapas: 1) recolección de muestras y análisis de las propiedades físicas y químicas del suelo, 2) análisis estadístico y descriptivo de las variables usando los software Statgraphic Centurión XVIII y Excel 2010, 3) análisis geoestadístico basado en la interpolación por el método Kriging con software GS+10, 4) análisis multivariado de las componentes principales generando un gráfico bidimensional (biplot) con InfoStat y 5) elaboración de mapas de variabilidad espacial de las propiedades físicas del suelo por medio de software Surfer 10 para la implementación del plan de cultivos y los mapas de uso del suelo, por medio de los software ArcGis 10. La infiltración del suelo resultó extremadamente heterogénea con un coeficiente de variación del 74,5\% y dependencia moderada con rango de alcance de $610,9 \mathrm{~m}$. Se delimitaron tres sectores para los cultivos mencionados, basados en los análisis del suelo según el uso potencial del suelo.
\end{abstract}

Palabras clave: Variabilidad espacial del suelo; propiedades físicas del suelo; uso del suelo.

\begin{abstract}
We studied the spatial variability of physical soil properties associated to sugar cane, grass and moringa crops in "Fluventic Ustropepts" soil of the lower basin of the Las Ceibas River. We used a 50m x 50m sampling grid in 26 points for the physical analysis, 8 infiltration tests using simple random sampling, and 3 integrated samples for chemical analysis in an area of 6.5 ha. The study was undertaken in five stages: 1 ) sample collection and analysis of the soil's physical and chemical properties; 2) statistical and descriptive analysis of the
\end{abstract}

[1] Ingeniera Agrícola. Universidad Surcolombiana- Neiva. Av. Pastrana Cra 1ª rociovargassilva@gmail.com

[2] Ingeniero Agrícola. Universidad Surcolombiana- Neiva. Av. Pastrana Cra 1ª ferchoserrato3@hotmail.com

[3] Ph.D. Ciencias Agropecuarias, Docente Universidad Surcolombiana-Neiva. Av. Pastrana Cra 1a. armando.torrente@gmail.com

Recibido: 22 febrero 2015 • Aceptado: 26 mayo 2015 
variables using Statgraphic Centurión XVIII software and Excel 2010; 3) geostatistical analysis based on Kriging interpolation using GS+10 software; 4) multivariate analysis of the main components leading to a bidimentional graph (biplot) using InfoStat; and 5) elaboration of spatial variability maps of the physical properties of soil using Surfer 10 software for the implementation of crop planning and soil mapping using ArcGis 10 software. Soil infiltration was extremely homogeneous with a $74.5 \%$ coefficient of variation of and moderate dependence with a working range of $610.9 \mathrm{~m}$. Three sectors were determined for the aforementioned crops based on the soil analysis for potential soil use.

Key words: spatial variation in soil, physical properties of soil, soil use.

\section{Introducción}

A inicios de la década del 90, inició el desarrollo de tecnologías de variabilidad espacial y temporal del suelo asociada con los aspectos de la producción agrícola, para mejorar los rendimientos y preservar la calidad ambiental. La variabilidad debe conocerse y ser de suficiente magnitud para que un manejo diferenciado por sitio resulte en un beneficio frente al manejo uniforme. Lozano et al. 2004, demostraron que aun a nivel de pequeñas parcelas experimentales, la variabilidad puede ser significativa. La variabilidad depende de la propiedad que se analice, siendo más variables las propiedades químicas que las físicas. Además, hay menor variabilidad en las propiedades del suelo, en su condición natural, que cuando es sometido a uso. Aquellas propiedades que más se afectan por el manejo del suelo serán las que presenten la mayor variabilidad. Además se ha comprobado que la variación en las propiedades del suelo puede deberse a diferencias regionales, como son clima, topografía y material parental (Mulla et al, 2002).

Las variaciones espaciales del suelo se pueden estudiar a través de técnicas geoestadísticas que permitirán elaborar mapas y así delimitar áreas de manejo diferencial. "Los suelos son cuerpos variables aún en cortas distancias, esta variabilidad es producto de las interacciones entre factores y procesos formadores de suelos" (Andriotti, 2010). Se ha estudiado la variabilidad de propiedades físicas, químicas y biológicas del suelo que afectan la productividad de los cultivos, reportándose asociaciones entre ellas y el rendimiento, aunque dichas asociaciones dependen del rango de variación del parámetro y del rango en que éste afecta al rendimiento, lo que permite medir y manejar la variabilidad espacial para aumentar la eficiencia productiva y disminuir el impacto ambiental.

Las propiedades físicas deben ser entendidas en conjunto y formando un todo armónico ya que entre ellas se establece una íntima relación. Estas propiedades de acuerdo a su determinación y con sus fenómenos relacionados, pueden ser divididas en dos grupos: físicas fundamentales que corresponden al color, la textura, estructura, la consistencia, la densidad y la temperatura, y físicas derivadas como la porosidad, la capacidad de aire, la capacidad de agua, la compactación y la profundidad efectiva radical (Torrente, 2013). Desde el punto de vista de su naturaleza, los atributos del suelo han sido agrupados en dos grandes categorías: variables estáticas y variables dinámicas. En física de suelos estos grupos han sido ampliamente estudiados (Ovalles, 2001) y se ha establecido que las variables estáticas tienen características que están influenciadas principalmente por la rígida matriz del suelo; con base en sus características se agrupan en propiedades estructurales, tales como la porosidad y la densidad aparente.

Los suelos del área de estudio presentan deficiencias en la mayoría de las características evaluadas, lo que hace que su explotación sea difícil y costosa. Las limitaciones más notorias son la deficiencia de nutrientes y la compactación del suelo. Se desea implementar los cultivos de caña, pastos y moringa para alimentación de ganado bajo un manejo adecuado de suelos, buscando indicadores para establecer las soluciones frente al déficit de nutrientes y los problemas de compactación del suelo, siendo importante la adopción de prácticas agrícolas sostenibles con aplicación de abonos orgánicos preferencialmente, ya que con ellos se pretende mejorar las propiedades físicas, químicas y biológicas del suelo.

Para implementar los cultivos de caña, pastos y moringa es necesario adaptar un plan de manejo ambiental basado en mapas de variabilidad espacial del suelo teniendo en cuenta zonas específicas de deficiencias, aplicando las enmiendas y prácticas necesarias para el mejoramiento de las condiciones del medio donde se desarrollará el cultivo. Lo anterior, se hace con miras 
a un buen desarrollo, crecimiento y productividad del cultivo desde el punto de vista sostenible, con un uso racional del suelo, el agua y el medio ambiente teniendo en cuenta que el plan de manejo ambiental debe ser a largo plazo y disponible a ajustes de acuerdo a las necesidades de los cultivos. En la presente investigación se pretende orientar la implementación, basados en el diagnóstico físico-químico del suelo, buscando la optimización de los cultivos apoyados en modelos geoestadísticos, con el fin de mejorar las prácticas de manejo del suelo.

\section{Metodología}

\subsection{Localización}

La finca Marandua-Venadito se localiza sobre una planicie fluvial a $530 \mathrm{~m}$ de altitud en el corregimiento de Fortalecillas del municipio de Neiva-Huila, sobre la margen derecha del rio Las Ceibas, con temperatura media de $28^{\circ} \mathrm{C}$, precipitación anual de $1350 \mathrm{~mm}$ y zona de vida bosque seco tropical según Holdridge en una extensión de 6.5 ha dedicadas esencialmente a potreros para ganadería bajo siembra de pastos. El área se ubica sobre una vega fluvial con acumulación de arenas de diverso tamaño y depósitos minerales diferenciales menos gruesos en profundidad en un horizonte superficial fuertemente compactado con dominio de texturas franco arenoso y arenoso franco (figura 1).

\subsection{Metodología en campo y laboratorio}

Se trazó una grilla de 50 m x 50 m sobre el mapa base del área, georeferenciando una red de puntos en campo con un receptor Garmin GPSmap-62sc e identificados mediante estacas. Se seleccionaron en total 26 puntos de muestreo físico del suelo 8 puntos para infiltración en un muestreo aleatorio simple. Se analizaron las propiedades físicas del suelo (textura, retención de humedad, densidad real, densidad aparente y porosidad), también se practicó muestreo representativo para la obtención de tres análisis químico del suelo siendo realizadas las pruebas en el Laboratorio de suelos - LABGAA de la Universidad Surcolombiana (tabla 1).

\subsection{Análisis estadístico}

Se hizo un análisis exploratorio de datos mediante el software Statgraphics Centurion XVI-II en el estudio de variabilidad espacial de las propiedades físicas del suelo basados en la red de puntos seleccionados al azar y así determinar su distribución. Se aplicó el software InfoStat versión estudiantil para el análisis multivariado y determinar las variables explicativas de los componentes principales del suelo y se realizó luego el análisis geoestadístico con el software Gs+10 for Windows, para determinar la relación y dependencia de las variables estudiadas espacialmente, ajustando los modelos geoestadísticos en los semivariogramas que representan la mayor eficiencia de los datos observados en campo.

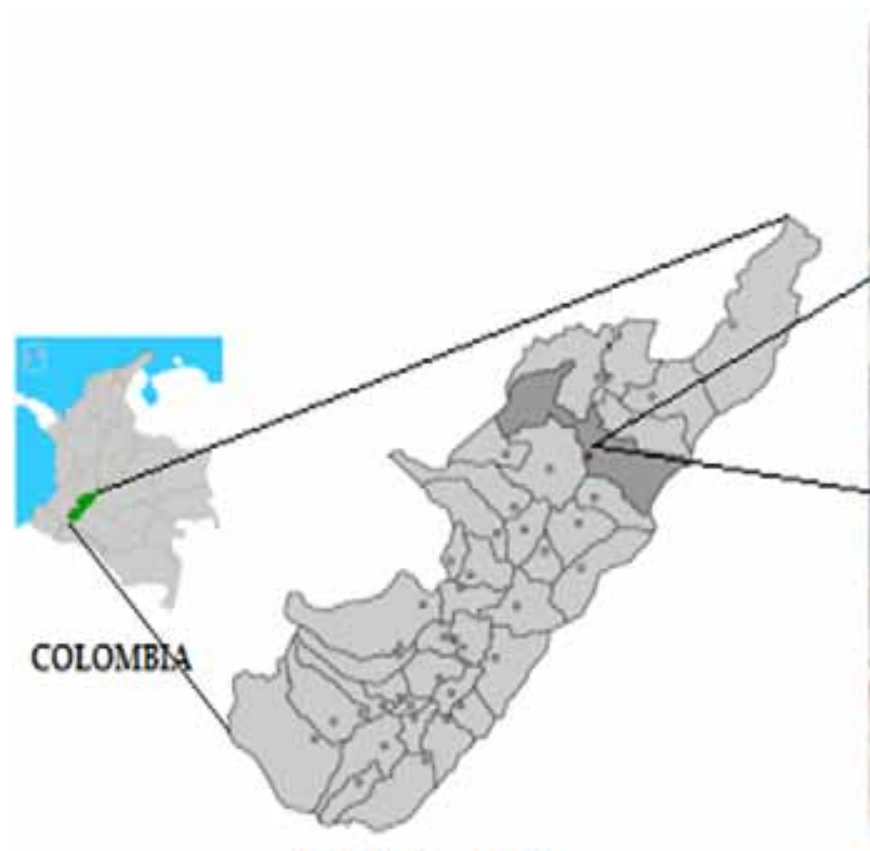

Fortalecillas Huila

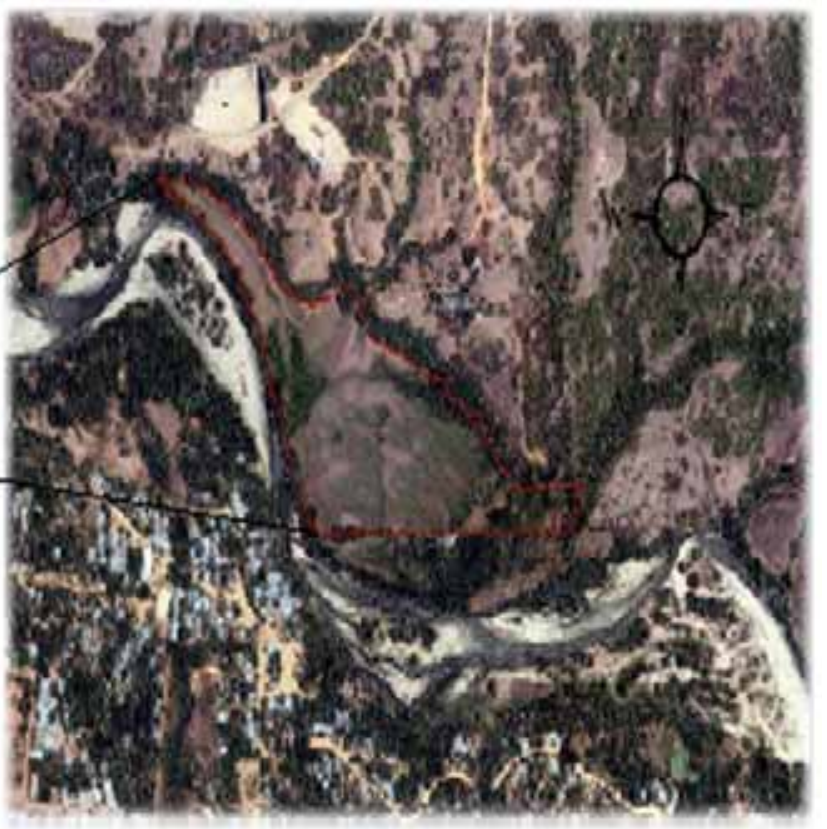

Area de Estudio

Figura 1. Localización del área de estudio en el departamento del Huila 
Tabla 1. Métodos en las propiedades físicas y químicas de los suelos evaluados.

\begin{tabular}{|c|c|c|c|c|c|}
\hline \multicolumn{3}{|c|}{ Propiedades físicas } & \multicolumn{3}{|c|}{ Propiedades químicas } \\
\hline Parámetro & Método & $\begin{array}{l}\text { Tamaño } \\
\text { muestra }\end{array}$ & Parámetro & Método & $\begin{array}{l}\text { Tamaño } \\
\text { muestra }\end{array}$ \\
\hline Textura & Bouyoucos & $30^{(1)}$ & $\mathrm{pH}$ & Potenciométrico & 3 \\
\hline Humedad a 0.3 bar & Membranas de Richard & $30^{(1)}$ & $\mathrm{C}$ & Walkley and Black & 3 \\
\hline Humedad a 15 bar & Membranas de Richard & $30^{(1)}$ & $\mathrm{CIC}$ & $\begin{array}{l}\mathrm{NH}_{4} \mathrm{OAc}, \mathrm{pH} 7,1 \\
\mathrm{~N}\end{array}$ & 3 \\
\hline Densidad aparente & Terrón parafinado & $30^{(1)}$ & $\begin{array}{l}\mathrm{Fe}, \mathrm{Mn}, \mathrm{Cu}, \\
\mathrm{Zn}\end{array}$ & Doble acido AA & 3 \\
\hline Densidad real & Picnómetro & $30^{(1)}$ & $\mathrm{S}$ & Turbidimetria & 3 \\
\hline Porosidad & Relación de densidades & $30^{(1)}$ & $\mathrm{B}$ & Agua Caliente & 3 \\
\hline Ks & Pozo barrenado inverso & 26 & $\mathrm{~K}, \mathrm{Ca}, \mathrm{Mg}, \mathrm{Na}$ & Absorción atómica & 3 \\
\hline Infiltración $^{(2)}$ & Anillos concéntricos & 8 & $\mathrm{P}$ & Bray II & 3 \\
\hline
\end{tabular}

Ks Conductividad hidráulica saturada.

${ }^{(1)}$ Incluye 4 muestras tomadas en calicata: ${ }^{(2)}$ Modelo de Kostiakov.

Metodología analítica en propiedades físicas y químicas del suelo: IGAC, 2006.

\section{Resultados y discusión}

\subsection{Descripción del Suelo}

El suelo posee un perfil diagnóstico caracterizado por horizonte superficial Ócrico y horizonte subsuperficial Cámbico con régimen de humedad Ustico y régimen de temperatura Isohipertérmico, siendo clasificado según USDA 2006 como Fluventic Ustropepts, localizado en posición de vega sobre relieve plano en la subcuenca baja del Rio Las Ceibas a una distancia de 100 m del cauce natural (tabla 2). La compactación del suelo es debida a los antecedentes asociados a prácticas de manejo tradicional en cultivo de arroz mecanizado y posteriormente a la introducción de ganadería bajo pastoreo, siendo este último el uso actual.

Tabla 2. Caracterización de las propiedades físicas del perfil de suelo.

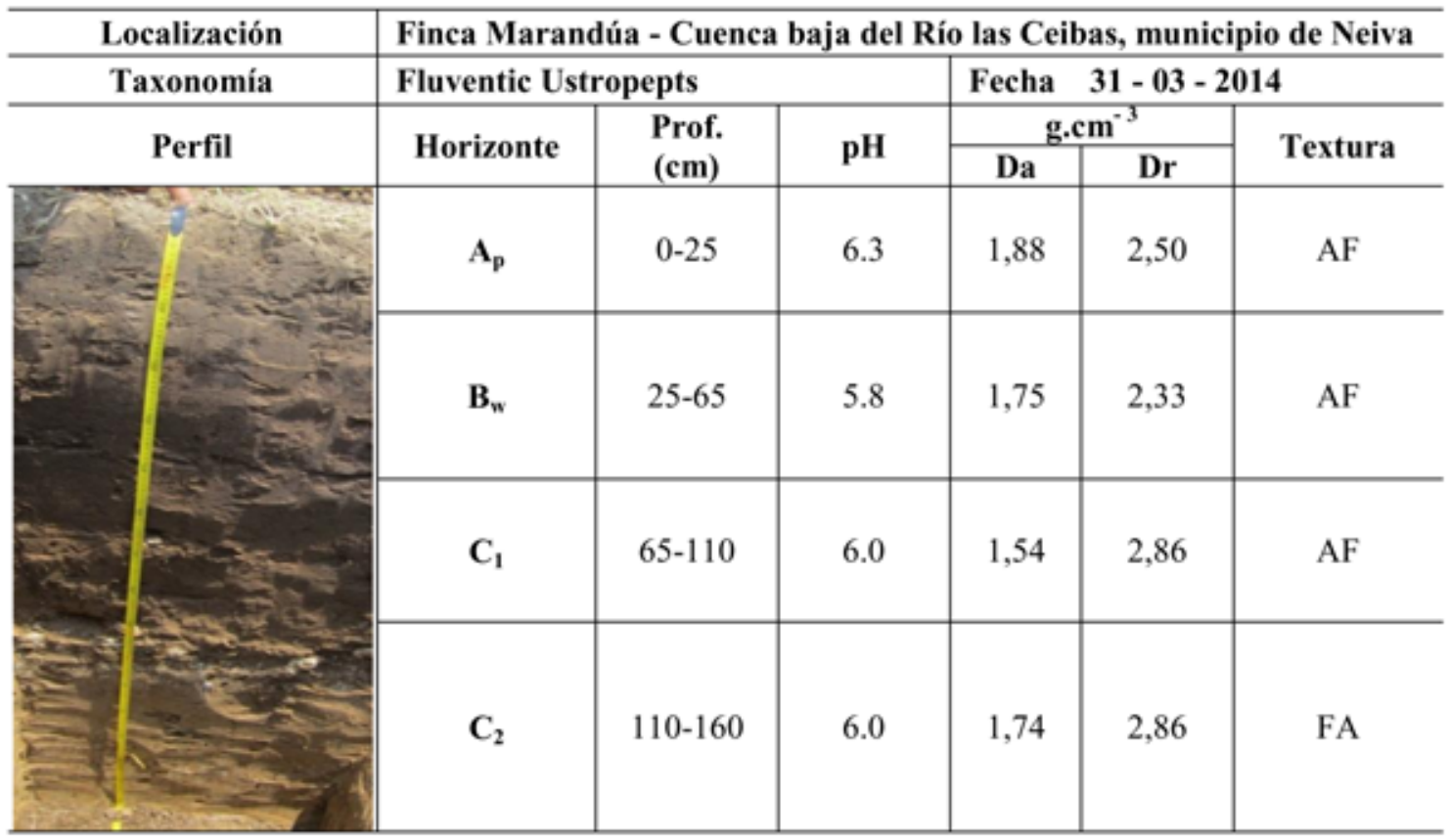

Da: densidad aparente, Dr: densidad real, AF: arenoso franco, FA franco arenoso 


\subsection{Propiedades físicas del Suelo}

La infiltración básica varió de moderadamente lenta a moderada $(0,23-3,87 \mathrm{~cm} / \mathrm{h})$ teniendo dominancia la infiltración moderadamente lenta, así mismo la conductividad hidráulica saturada del suelo resultó entre moderadamente lenta y moderada (1,22 y 1,99 m/ día), siendo afectada estas propiedades hidrodinámicas por la compactación en los primeros $25 \mathrm{~cm}$ de profundidad del suelo (tablas 3).

Las propiedades físicas del suelo mediante el análisis univariado se clasificaron considerando el coeficiente de variación estadístico (CV) en 4 categorías definidas por Vásquez 2009, así (tabla 4).

Da es la densidad aparente, Dr es la densidad real, $\mathrm{h}$ es la porosidad total, Ks es la conductividad hidráulica saturada, CC es la humedad gravimétrica a la capacidad de campo, PMP es la humedad gravimétrica al punto de marchitez permanente, A es arena, Ar es arcilla, L es limo, I es la infiltración del suelo.

La densidad aparente del suelo varió ampliamente entre 1,27 y $2,0 \mathrm{~g} / \mathrm{cm}^{3}$, siendo afectada por procesos de compactación debido a los antecedentes de mecanización en el monocultivo de arroz y el pastoreo durante los últimos años. La densidad real corresponde a suelos minerales con bajos contenidos de materia orgánica.

El suelo es de textura arenoso franco a franco arenoso con inclusiones localizadas de textura arenosa, siendo la arena, la fracción mineral dominante cuya variación está entre 74,28 y 88,06\%, teniendo en correspondencia tendencia a la baja porosidad, esto explica la baja infiltración y escasa capacidad de retención de humedad en el suelo (Burbano, 2010). La disponibilidad del agua en el suelo varió ampliamente con muy baja capacidad de retención de agua en la mayoría de los sitios con valores entre 8-15\%, requiriendo altas frecuencias de riego para el mantenimiento de los contenidos de humedad adecuados en el suelo para el desarrollo normal de los cultivos (tabla 5).

Obsérvese la alta variabilidad de la infiltración del suelo relacionada con la clase textural arenosa $(\mathrm{CV}=$ 4.23) y su afectación por los problemas de compactación, así mismo la densidad aparente y la porosidad total del suelo muestran los efectos anotados como

Tabla 3. Clasificación de la infiltración del suelo.

\begin{tabular}{c|c|c|c|l|c|c}
\hline \multirow{2}{*}{$\begin{array}{c}\text { Función infiltración } \\
\text { acumulada }\end{array}$} & $\begin{array}{c}\mathbf{T}_{\mathbf{b}} \\
\mathbf{I}(\mathbf{c m}), \mathbf{t}(\mathbf{m i n})\end{array}$ & $\begin{array}{c}\mathbf{I}_{\mathbf{b}} \\
(\mathbf{m i n})\end{array}$ & \multicolumn{2}{|c|}{ Clase } & \multicolumn{2}{c}{ Coordenadas planas $(\mathbf{m})$} \\
\cline { 5 - 7 } & & & & Este & Norte \\
\hline 2 & $I=5.36\left(t^{0.349}\right)$ & 390.18 & 0.23 & Lenta & 870326.589 & 818210.154 \\
\hline 3 & $I=2.42\left(t^{0.780}\right)$ & 131.88 & 3.87 & Moderada & 870333.224 & 818136.430 \\
\hline 4 & $I=1.84\left(t^{0.608}\right)$ & 234.84 & 0.79 & Moderada lenta & 870208.698 & 818149.833 \\
\hline 5 & $I=2.07\left(t^{0.683}\right)$ & 142.56 & 1.67 & Moderada lenta & 870474.861 & 818108.105 \\
\hline 6 & $I=2.19\left(t^{0.490}\right)$ & 305.58 & 0.34 & Lenta & 870139.911 & 818297.003 \\
\hline 7 & $I=3.16\left(t^{0.068}\right)$ & 188.34 & 2.51 & Moderada & 870065.477 & 818361.230 \\
\hline 8 & $I=2.86\left(t^{0.663}\right)$ & 202.14 & 1.90 & Moderada lenta & 869992.137 & 818407.759 \\
\hline
\end{tabular}

$\mathrm{I}$ : es la infiltración acumulada, $\mathrm{T}_{\mathrm{b}}$ : es el tiempo básico, $\mathrm{I}_{\mathrm{b}}$ : es la infiltración básica del suelo

Tabla 4. Clasificación de las variables físicas del suelo según el coeficiente de variación CV.

\begin{tabular}{c|l|l}
\hline CV\% & \multicolumn{1}{|c}{ Clasificación de las variables } & \multicolumn{1}{c}{$\begin{array}{c}\text { Variables físicas } \\
\text { del suelo }\end{array}$} \\
\hline$<20$ & Relativamente homogéneas & Da, Dr, A, Ar, Ks \\
\hline $20-40$ & Moderadamente homogéneas & PMP, L \\
\hline $40-60$ & Normalmente heterogéneas & $\mathrm{CC}, \eta$ \\
\hline$>60$ & Extremadamente heterogéneas & $\mathrm{I}$ \\
\hline
\end{tabular}


Tabla 5. Resumen estadístico de las propiedades físicas del suelo.

\begin{tabular}{c|c|c|c|c|c|c|c|c|c|c}
\hline Variable & $\bar{X}$ & $\mathbf{M e}$ & $\mathbf{S}^{\mathbf{2}}$ & $\mathbf{S}$ & $\begin{array}{c}\mathbf{\%} \\
\mathbf{C V}\end{array}$ & $\mathbf{M i n}$ & $\mathbf{M a x}$ & Rango & Sesgo & Curtosis \\
\hline $\mathbf{D}_{\mathbf{a}}$ & 1,83 & 1,81 & 0,08 & 0,29 & 15,90 & 1,27 & 2,00 & 1,02 & 0,13 & $-1,04$ \\
\hline $\mathbf{D}_{\mathbf{r}}$ & 2,56 & 2,82 & 0,14 & 0,37 & 14,42 & 2.12 & 2,86 & 1,04 & $-1,67$ & $-0,83$ \\
\hline $\mathbf{C C}$ & 12,89 & 11,69 & 28,83 & 5,37 & 41,66 & 5,10 & 28,98 & 23,88 & 2,34 & 1,80 \\
\hline $\mathbf{P M P}$ & 4,68 & 4,68 & 1,26 & 1,12 & 23,99 & 1,67 & 6,62 & 4,95 & $-0,78$ & 0,87 \\
\hline $\mathbf{A}$ & 80,58 & 80,82 & 11,63 & 3,41 & 4,23 & 74,28 & 88,06 & 13,78 & 0,25 & $-0,24$ \\
\hline $\mathbf{L}$ & 9,51 & 9,89 & 3,96 & 1,99 & 20,91 & 5,34 & 13,08 & 7,74 & $-0,42$ & $-0,58$ \\
\hline $\mathbf{A r}$ & 9,91 & 9,05 & 3,85 & 1,96 & 19,80 & 6,60 & 13,13 & 6,53 & 0,45 & $-1,04$ \\
\hline $\mathbf{K s}$ & 1,60 & 1,63 & 0,06 & 0,24 & 14,88 & 1,22 & 1,99 & 0,77 & $-0,22$ & $-1,11$ \\
\hline $\boldsymbol{\eta}$ & 27,72 & 23,43 & 204,84 & 14,31 & 51,63 & 1,72 & 51,48 & 49,76 & $-0,09$ & $-1,21$ \\
\hline $\mathbf{I}$ & 1,62 & 1,64 & 1,46 & 1,21 & 74,55 & 0,23 & 3,88 & 3,65 & 0,88 & 0,29 \\
\hline $\mathbf{X}$
\end{tabular}

X: Media, Me Mediana, $S^{2}$ : Varianza, S Desviación estándar, CV: coeficiente de variación, Min: Mínimo, Max: Máximo, $\mathrm{D}_{\mathrm{a}}$ : Densidad aparente, $\mathrm{D}_{\mathrm{r}}$ densidad real, CC: capacidad de campo y PMP: punto de marchitez permanente, A: arena, L: limo, Ar: arcilla, conductividad hidráulica saturada (m/día), $\eta$ : porosidad total, I: infiltración $(\mathrm{cm} / \mathrm{h})$.

consecuencia del manejo antecedente del suelo. La conductividad hidráulica saturada resultó con ligera variabilidad a diferencia de otros estudios (Torrente 2003, Giraldo y Mendoza 2003, Gutiérrez y Castaño, 2013), que demuestran que esta propiedad hidrodinámica es la que presenta el mayor rango de variación, con coeficientes que llegan a superar el 100\%.

\subsection{Propiedades químicas del suelo.}

Se realizó el muestreo de suelos en tres sectores del área de estudio para el análisis de fertilidad mostrando que los suelos son ligeramente ácidos con muy bajos contenidos de materia orgánica, baja capacidad de intercambio catiónico, alta saturación de bases, bajos contenido de fósforo y azufre con deficiencias en cobre y cinc. Para el plan de cultivos que se desea implementar debe de considerarse la aplicación de abonos orgánicos minerales con el fin de suplir las deficiencias nutricionales y mejorar las características físicas relacionadas con retención de agua y nutrientes para las plantas (tabla 6).

\subsection{Modelos de semivariogramas.}

Los modelos de los semivariogramas seleccionados corresponden a los de mayor ajuste espacial que explican la distribución de la variable física evaluada. Se observa en la tabla 7, que los semivariogramas con el modelo lineal representan las variables separados del suelo (arena, limo y arcilla), así mismo el coeficiente de punto de marchitez permanente, la porosidad total e infiltración se ajustan al modelo exponencial. Por otro lado, las variables coeficiente de capacidad de campo, densidad aparente y la conductividad hidráulica se ajustan a modelos esféricos.

La dependencia espacial se analizó por medio del efecto Nugget relativo $\left[\% \mathrm{C}_{0} /\left(\mathrm{C}_{0}+\mathrm{C}\right)\right.$ ], clasificación según Cambardella et al. 1994, citado por Trujillo 2011, resultando fuerte dependencia para las variables densidad aparente $\left(D_{\mathrm{a}}\right)$, densidad real $\left(\mathrm{D}_{\mathrm{r}}\right)$, capacidad de campo (CC), porosidad y conductividad hidráulica saturada del suelo ( $<25 \%)$, mientras que punto de marchitez permanente e infiltración tienen una dependencia moderada (25-75\%) y débil dependencia espacial (> 75\%) para arena, limo y arcilla. La variable física con mayor alcance de dependencia espacial $\left(A_{0}\right.$ ) fue la infiltración con 610,9 $\mathrm{m}$ y la de menor dependencia fue la porosidad total del suelo con $30.5 \mathrm{~m}$, lo que significa que el muestreo de suelos para la variable infiltración del suelo correlaciona a distancias mayores respecto a las otras variables evaluadas (tabla 6).

El coeficiente de Pearson permitió analizar la relación entre las distintas variables físicas del suelo, resultado una relación proporcional directa para las variables de capacidad de campo (CC) y arcilla $(0,51)$, densidad real y porosidad total $(0,65)$; mientras que relación inversa para las variables densidad aparente y porosidad $(-0,70)$, capacidad de campo (CC) y arena $(-0,55)$, arena y limo $(-0,87)$, arena y arcilla $(-0,86)$ (tabla 8$)$. 
Tabla 6. Propiedades químicas del suelo en tres sectores del área.

\begin{tabular}{|c|c|c|c|c|c|}
\hline Parametros quimicos & Unidad & $\begin{array}{c}\text { Sector } 1 \\
\text { Pasto } \\
\text { Tanzania }\end{array}$ & $\begin{array}{l}\text { Sector } 2 \\
\text { Moringa }\end{array}$ & $\begin{array}{c}\text { Sector } 3 \\
\text { Caña }\end{array}$ & Método \\
\hline $\mathrm{pH}$ & - & 6.3 & 6.2 & 6.3 & NTC 5264 \\
\hline Carbono Orgánico (CO) & $\%$ & 0.61 & 0.70 & 0.58 & $\begin{array}{l}\text { NTC } 5403 \\
\text { método B }\end{array}$ \\
\hline C.I.C. & $\mathrm{cmol}^{+} \cdot \mathrm{kg}^{-1}$ & 16.1 & 11.3 & 12.9 & NTC 5268 \\
\hline Fósforo $(\mathrm{P})$ & ppm & 40.2 & 6.44 & 12.8 & NTC 5350 \\
\hline Calcio $(\mathrm{Ca})$ & $\mathrm{cmol}^{+} \cdot \mathrm{kg}^{-1}$ & 12.76 & 7.91 & 9.00 & \multirow{4}{*}{ NTC 5349} \\
\hline Magnesio (Mg) & $\mathrm{cmol}^{+} \cdot \mathrm{kg}^{-1}$ & 3.67 & 2.26 & 2.73 & \\
\hline Sodio $(\mathrm{Na})$ & $\mathrm{cmol}^{+} . \mathrm{kg}^{-1}$ & 0.33 & 0.25 & 0.33 & \\
\hline Potasio $(\mathrm{K})$ & $\mathrm{cmol}^{+} \cdot \mathrm{kg}^{-1}$ & 0.32 & 0.21 & 0.19 & \\
\hline Bases Totales (BT) & $\mathrm{cmol}^{+} \cdot \mathrm{kg}^{-1}$ & 17.1 & 10.6 & 12.3 & Suma cationes \\
\hline $\begin{array}{l}\text { Saturación de bases } \\
\text { (SB) }\end{array}$ & $\%$ & $>100$ & 94.1 & 94.9 & $\begin{array}{l}\text { Relación } \\
\text { catiónica }\end{array}$ \\
\hline Azufre (S) & ppm & 4.47 & 3.26 & 3.25 & NTC 5402 \\
\hline Hierro (Fe) & ppm & 86.8 & 84.4 & 88.6 & \multirow{4}{*}{$\begin{array}{l}\text { NTC } 5526 \\
\text { método DTPA }\end{array}$} \\
\hline Manganeso (Mn) & ppm & 27.6 & 31.6 & 29.5 & \\
\hline Cobre $(\mathrm{Cu})$ & $\mathrm{ppm}$ & 2.11 & 1.67 & 1.71 & \\
\hline Cinc (Zn) & ppm & 1.37 & 0.89 & 0.86 & \\
\hline Boro (B) & ppm & 0.20 & 0.20 & 0.31 & $\mathrm{H}_{2} \mathrm{O}$ Caliente \\
\hline Acidez intercambiable & $\mathrm{cmol}^{+} \cdot \mathrm{kg}^{-1}$ & - & - & - & NTC 5263 \\
\hline Relación $\mathrm{Ca} / \mathrm{Mg}$ & - & 3.48 & 3.50 & 3.30 & \multirow{3}{*}{$\begin{array}{l}\text { Relación } \\
\text { catiónica }\end{array}$} \\
\hline Relación $(\mathrm{Ca}+\mathrm{Mg}) / \mathrm{K}$ & - & 51.34 & 48.4 & 61.7 & \\
\hline Relación $\mathrm{Mg} / \mathrm{K}$ & - & 11.5 & 10.8 & 14.4 & \\
\hline Textura & Clase & FA & FA & FA & Organoléptico \\
\hline
\end{tabular}

Tabla 7. Parámetros de los semivariogramas para las propiedades físicas del suelo.

\begin{tabular}{c|l|c|c|c|c|c|c}
\hline Variable & Modelo & $\begin{array}{c}\text { Nuguet } \\
(\mathbf{C o})\end{array}$ & $\begin{array}{c}\text { Sill } \\
(\mathbf{C o}+\mathbf{C})\end{array}$ & $\begin{array}{c}\mathbf{\%} \mathbf{C o} / \\
(\mathbf{C o}+\mathbf{C})\end{array}$ & $\begin{array}{c}\text { Rango } \\
(\mathbf{A o})\end{array}$ & $\mathbf{R}^{\mathbf{2}}$ & Dependencia \\
\hline $\mathrm{Da}$ & Esférico & 0.002 & 0.087 & 3.09 & 105.4 & 0.756 & Fuerte \\
\hline $\mathrm{Dr}$ & Gaussiano & 0.100 & 0.452 & 22.12 & 185.0 & 0.761 & Fuerte \\
\hline $\mathrm{CC}$ & Esférico & 30.370 & 247.2 & 12.28 & 88.6 & 0.188 & Fuerte \\
\hline$\eta$ & Exponencial & 24.00 & 216.9 & 11.06 & 30.5 & 0.132 & Fuerte \\
\hline $\mathrm{Ks}$ & Esférico & 0.005 & 0.062 & 9.13 & 93.4 & 0.251 & Fuerte \\
\hline $\mathrm{PMP}$ & Exponencial & 0.457 & 1.726 & 26.47 & 121.9 & 0.926 & Moderada \\
\hline $\mathrm{I}$ & Exponencial & 1.127 & 3.505 & 32.15 & 610.9 & 0.427 & Moderada \\
\hline $\mathrm{A}$ & Lineal & 11.464 & 11.464 & 100 & 258.7 & 0.472 & Débil \\
\hline $\mathrm{L}$ & Lineal & 3.926 & 3.926 & 100 & 258.7 & 0.940 & Débil \\
\hline $\mathrm{Ar}$ & Lineal & 0.039 & 0.039 & 100 & 258.7 & 0.841 & Débil \\
\hline
\end{tabular}

Da: densidad aparente, Dr: densidad real, CC: capacidad de campo, $\eta$ porosidad total, Ks: conductividad hidráulica saturada, PMP: punto de marchitez permanente, I: infiltración básica, A: arena, L: limo, Ar: arcilla.

\subsection{Análisis Multivariado}

cial del suelo desde sus componentes principales en virtud de una serie de variables explicativas halladas por medio del análisis multivariado a partir de las propiedades físicas del suelo. Se establecieron los valores de los autovectores de ponderación (e1, e2 y e3) con valor absoluto alrededor del $45 \%$ y un coeficiente de correlación de componentes principales (CP1, CP2 y CP3) mayor a 60\%. El contenido de arena (A), la densidad aparente $(\mathrm{Da})$ y la densidad real $(\mathrm{Dr})$ son las variables más explicativas de los componentes 1,2 y 3 
Tabla 8. Coeficiente de correlación - Pearson de las propiedades físicas del suelo.

\begin{tabular}{c|c|c|c|c|c|c|c|c|c}
\hline Variable & Da & Dr & CC & PMP & A & L & Ar & $\eta$ & Ks \\
\hline $\mathrm{Da}$ & 1.00 & & & & & & & & \\
\hline $\mathrm{Dr}$ & 0.07 & 1.00 & & & & & & & \\
\hline $\mathrm{CC}$ & -0.08 & -0.25 & 1.00 & & & & & & \\
\hline $\mathrm{PMP}$ & 0.14 & 0.06 & -0.15 & 1.00 & & & & & \\
\hline $\mathrm{A}$ & -0.20 & -0.04 & -0.55 & -0.32 & 1.00 & & & & \\
\hline $\mathrm{L}$ & 0.02 & 0.03 & 0.44 & 0.38 & -0.87 & 1.00 & & & \\
\hline $\mathrm{Ar}$ & 0.34 & 0.05 & 0.51 & 0.17 & -0.86 & 0.49 & 1.00 & & \\
\hline$\eta$ & -0.70 & 0.65 & -0.11 & -0.10 & 0.14 & -0.01 & -0.24 & 1.00 & \\
\hline $\mathrm{Ks}$ & -0.22 & 0.03 & 0.04 & -0.26 & 0.04 & -0.23 & 0.16 & 0.18 & 1.00 \\
\hline
\end{tabular}

pa: densidad aparente, pr: densidad real, CC: Capacidad de campo, PMP: punto de marchitez permanente, A: arena, L: limo, Ar: arcilla, $\eta$ : porosidad total, Ks: conductividad hidráulica saturada.

respectivamente, explicando así el 73\% de la variación de las propiedades físicas del suelo evaluadas.

El análisis estadístico a través del diagrama biplot es apreciable la relación directa entre la densidad real (Dr) y la porosidad total (h) y entre la humedad a capacidad de campo (CC) y el agua aprovechable (AA) del suelo, también es evidente la escasa correlación entre la densidad real (Dr) y los contenidos de arena (A) y limo (L) en el suelo; los componentes principales 2 y 3 representan el 20.5 y $18.2 \%$ de la variabilidad respectivamente, lo que confirma una correlación alta entre las variables inicialmente citadas.

\subsection{Variabilidad espacial del suelo}

Textura y densidad del suelo. En general el porcentaje de arena en el suelo es alto (74,28 - 88,06\%), con valores muy bajos de otros separados minerales restantes como son arcilla (6,6 - 13,13\%) y limo (5,34 -13,08\%), intensificándose la arena hacia el norte del área (84 - 88\%) siendo baja la capacidad de retención de humedad y disponibilidad de agua y nutrientes para las plantas (Torrente, 2010). La densidad real del suelo varió entre 2,45 y 2,89 gr/ $\mathrm{cm}^{3}$, mientras la densidad aparente con valores altos incrementó desde el noroeste hacia el sureste del área. Los altos valores de densidad aparente son consecuencia del uso, con incremento de la compactación que afecta en general las propiedades físicas del suelo (figura 2).

Agua disponible y porosidad total. El agua disponible presentó niveles muy bajos (1,67 -6,62\%), ajustados al grupo textural del suelo (Figura 3a). La porosidad total resultó muy baja a media (1,72 a 40\%) distribuidos en el 84,6\% del área, la porosidad media se localiza en la zona central con disminución hacia los extremos (Figura 3b).

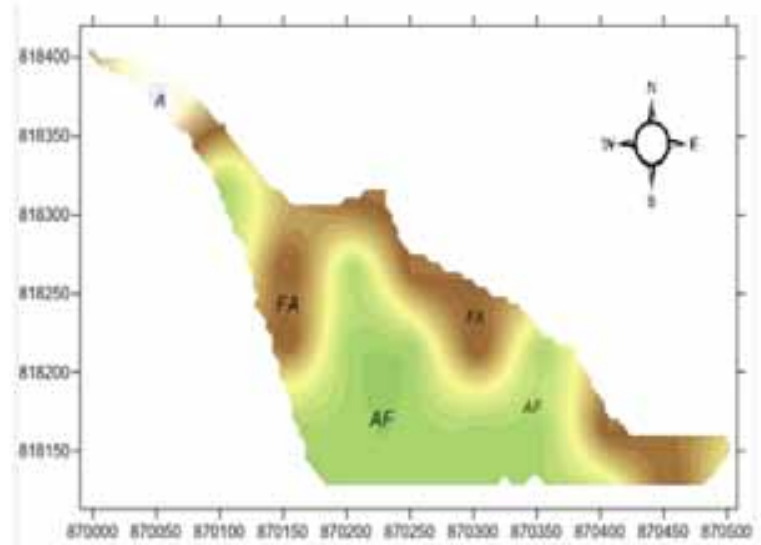

a)

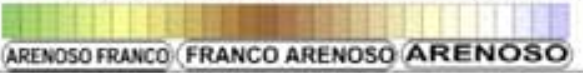

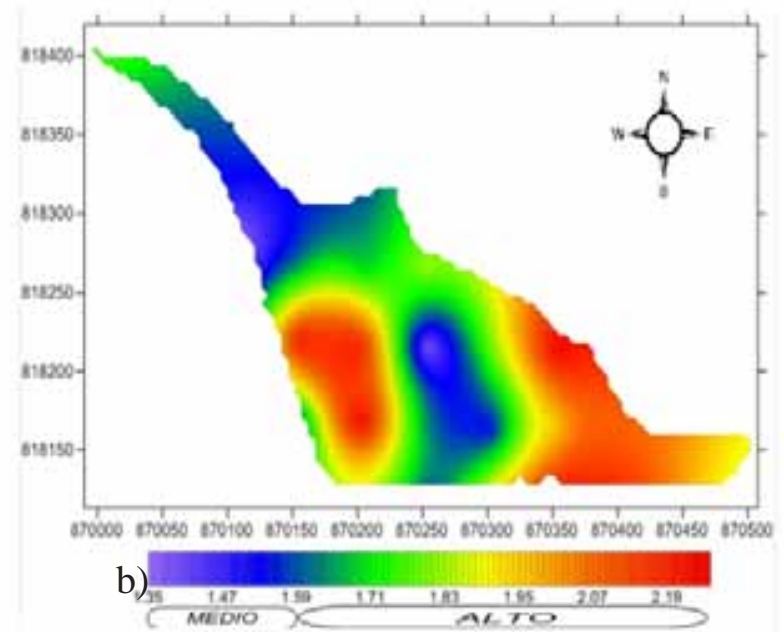

Figura 2. a) Mapa textural del suelo. b) Mapa de la densidad aparente. 


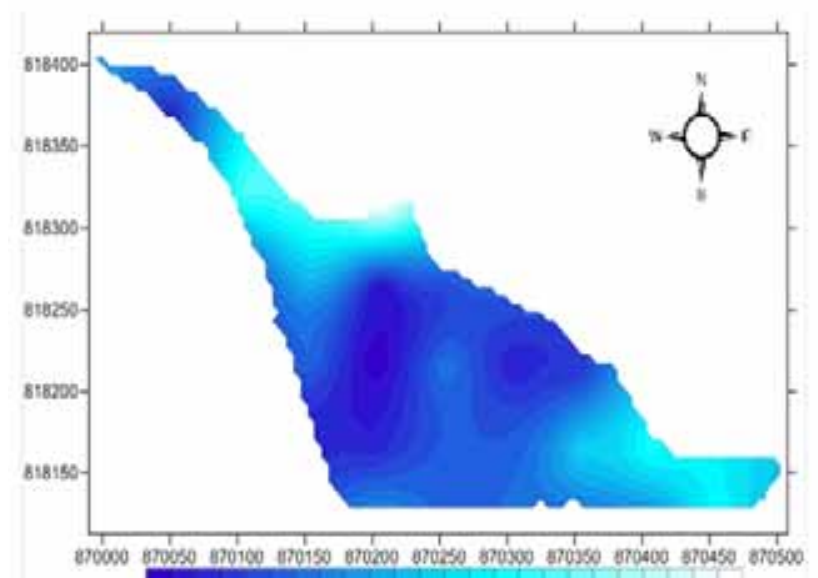

a)

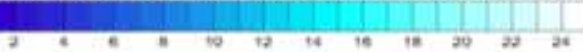

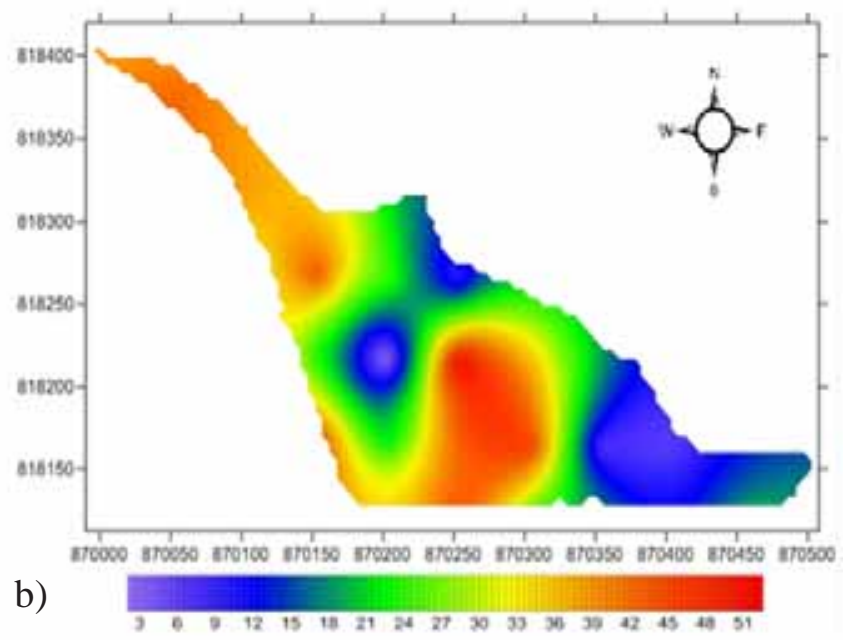

Figura 3. a) Mapa de agua disponible en el suelo. b) Mapa de Porosidad total del suelo.

Infiltración y conductividad hidráulica. La infiltración del agua en el suelo varió de lenta a moderada $(0,22-3,88 \mathrm{~cm} / \mathrm{h}$ (Figura $4 \mathrm{a})$ y la conductividad hidráulica del suelo varió de moderadamente lenta a moderada e igualmente está afectada por los procesos de compactación debido a los antecedentes de mecanización y pisoteo de ganado. La conductividad hidráulica incrementa desde el sureste hasta el noroeste, localizándose en este último sector los mayores valores (Figura $4 b$ ).

\subsection{Mapas de uso del suelo}

Uso actual. El área esta sectorizada en dos usos actuales, un sector dedicado a pastoreo libre del ganado
(5,82 ha) y otro sector con pastos de corte (0,68 ha) (Figura 5b).

Uso Potencial. Se consideraron los atributos químicos y físicos del suelo y las necesidades nutricionales de los cultivos a implementar (Figura 5a); por lo tanto se decidió establecer en la zona noroeste (2.23 ha) el cultivo de moringa, pues esta franja cuenta con niveles óptimos de fósforo y magnesio, elementos que junto al azufre participan activamente en la síntesis de aceites en plantas oleaginosas entre otras. Sin embargo "la mayor parte del P en la solución de suelo no es aprovechable por las plantas debido a su gran insolubilidad, por lo cual los cultivos responden positivamente a las aplicaciones de este elemento” (Navarro y García 2003).
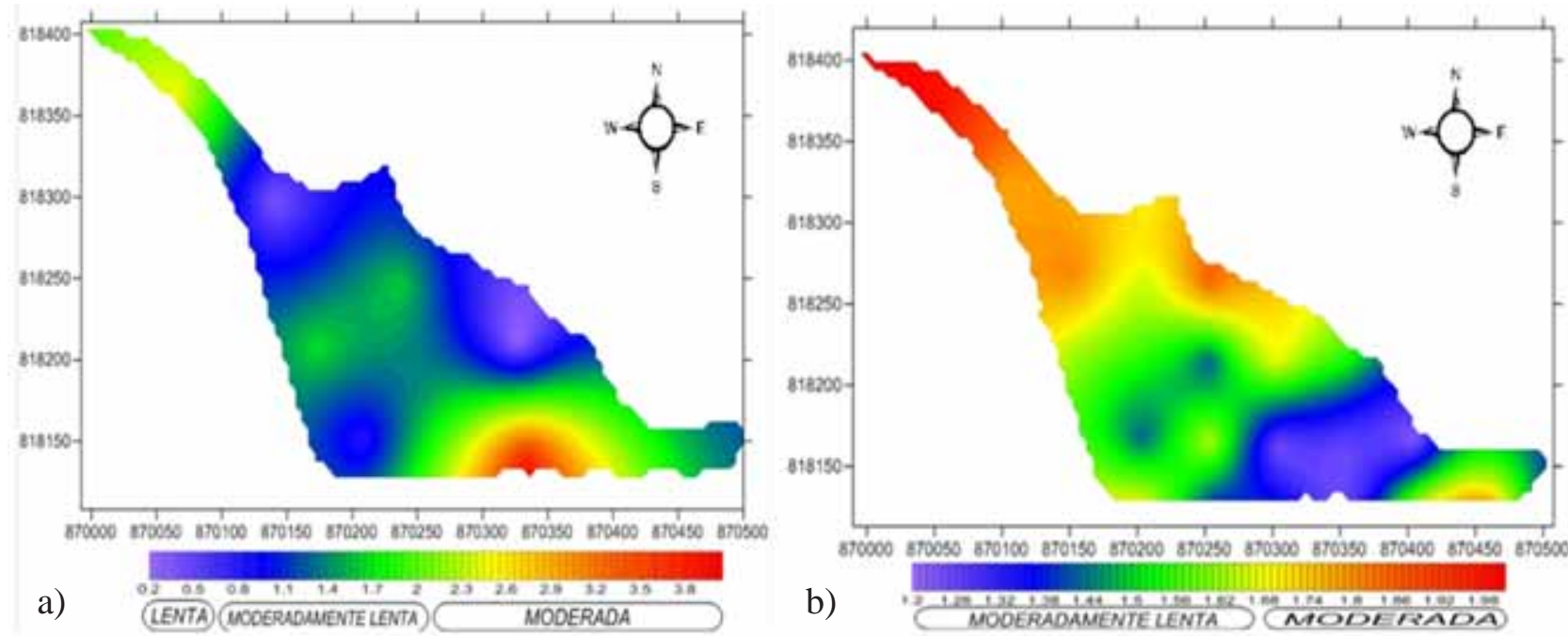

Figura 4. a) Mapa de infiltración y b) mapa de conductividad hidráulica saturada del suelo 


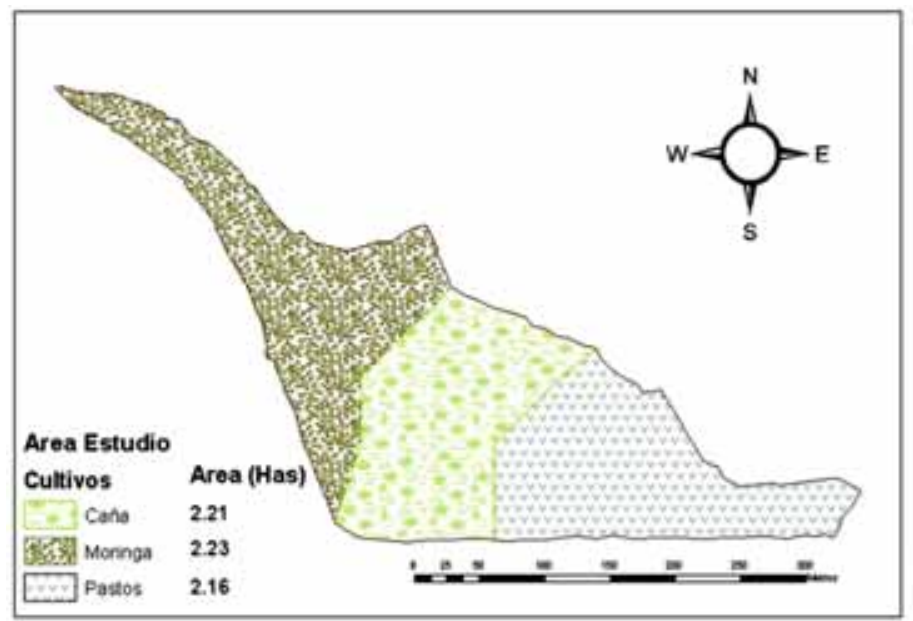

a)

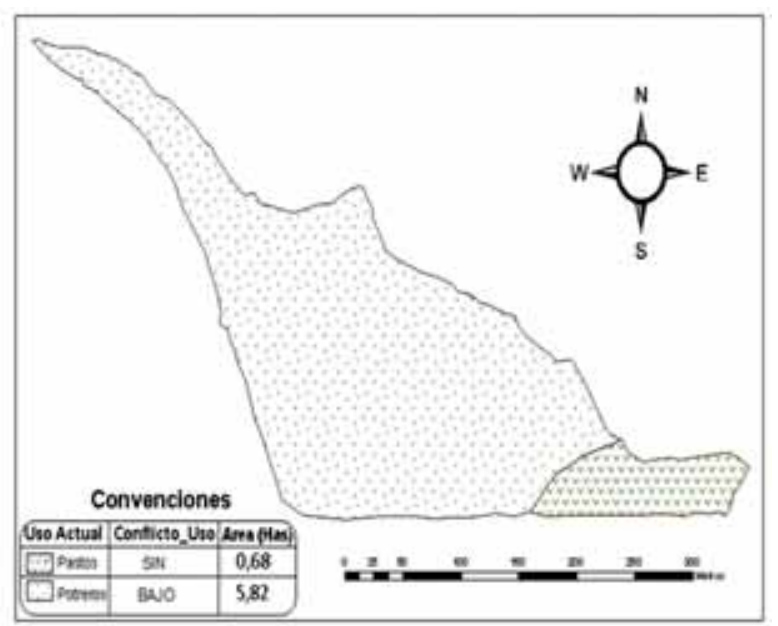

b)

Figura 5. a) Mapa de Uso potencial del suelo. b) Mapa de Uso actual y conflicto de uso del suelo.

Con base en las características físicas y químicas del suelo, el sureste del área (2,16 ha) es adecuado para pastos y en la actualidad se dedica a éste cultivo, mostrando rendimiento aceptable. Se requiere la aplicación de abonos orgánico-minerales para suplir las deficiencias nutricionales que favorezcan el desarrollo y los rendimientos del cultivo; es de resaltar que el $\mathrm{pH}$ del suelo es adecuado, ya que es cercano a la neutralidad. El cultivo de caña se recomienda implementar en la parte central del área (2.21 ha), la cual presenta déficits de fósforo (> $10 \mathrm{ppm})$ y de potasio $(<0.2 \mathrm{cmol} / \mathrm{kg})$, elementos a suplementar a través del plan de fertilización orgánico-mineral a sugerir por el ingeniero agrónomo (Figura 5b).

Conflicto de uso. El conflicto de uso se califica medio, ya que se cambia de potrero a cultivo, lo que no implica un cambio radical en el uso del suelo (Figura 5b).

\section{Conclusiones}

El análisis geoestadístico permitió conocer la variación de las propiedades físicas del suelo siendo la infiltración, la variable con mayor heterogeneidad con un coeficiente de variación de 54,5\%, mientras que la porosidad total y la capacidad de campo son variables normalmente heterogéneas.

La variable con mayor dependencia espacial fue la infiltración básica del suelo con 610,9 m mientras que la de menor dependencia espacial fue la porosidad total con $30,5 \mathrm{~m}$.

El suelo Fluventic Ustropepts posee densidad aparente alta con valores superiores a 1,6 g. $\mathrm{cm}^{-3}$, como consecuencia de antecedentes de uso y manejo del suelo asociado a la compactación de horizontes arenosos.

Las propiedades físicas del suelo directamente proporcionales son: porosidad total y densidad real, contenido de arcilla y humedad a la capacidad de campo.

Se determinó un conflicto medio del suelo en un $87 \%$ del área, cambiando de potreros a cultivos de moringa, caña y pastos.

\section{Referencias bibliográficas}

1. Andriotti, J.L.S. 2010. Fundamentos de Estadística e Geoestatística. São Leopoldo: Editora Unisinos. 165 p.

2. Burbano, H. 2010. Ciencia del Suelo. Principios Básicos. Sociedad Colombiana de la Ciencia del Suelo.

3. Giraldo, S. y A. M. Mendoza. 2003. Caracterización de la variabilidad espacial de las propiedades físicas de los lotes experimentales destinados al cultivo de hortalizas en Ceunp. Universidad del Valle, Facultad 
de Ingeniería, Santiago de Cali, Universidad Nacional de Colombia, Departamento de Ingeniería, Palmira. 112 p.

4. Gutiérrez, J.; I. Castaño. 2013. Variabilidad espacial de algunas propiedades del suelo con fines agropecuarios en la Finca La Primavera - Las Delicias municipio de Teruel, Huila. Tesis de grado, Programa de Ingeniería Agrícola, Universidad Surcolombiana. Neiva, Huila. 100 p.

5. IGAC, Instituto Geográfico Agustín Codazzi. 2006. Métodos analíticos de laboratorio de suelos. pp. 8-411.

6. Lozano, Z., Bravo, C., Ovalles, F., Hernández, M., Moreno, B., Piñango, L. y Villanueva, J. 2004. Selección de un diseño de muestreo en parcelas experimentales a partir del estudio de la variabilidad espacial de los suelos. Bioagro, 16(1), 61-72.

7. Mulla, D. Mcbratney, A. McBratney. 2002. "Soil Spatial Variability”. A.Warrick. (editor). Soil Physics Companion. Ed. CRC Press. Boca Raton, US. pp. 343-373.

8. Navarro, S., Ginés, B., García, N. 2003. Química Agrícola: el suelo y los elementos químicos esenciales para la vida $2^{\text {a }}$ edición. Madrid, España. pp. 233-246.

9. Ovalles, F. Memorias del curso de Geoestadística Aplicada a Estudios Ambientales con Énfasis en Suelos. Manizales: Universidad de Caldas 2001. 35 p.
10. Torrente T., A. 2003. Características físicas e hidrodinámicas en suelos con alta saturación de magnesio en el Valle del Cauca. Tesis Doctoral, Facultad de Ciencias Agropecuarias, Universidad Nacional de Colombia sede Palmira. Palmira, Valle del Cauca. 233 p.

11. Torrente T., A. y Valenzuela, I. 2010. Física de Suelos. Ciencia del Suelo Principios Básicos. Bogotá: Sociedad Colombiana de la Ciencia del Suelo, p. 65.

12. Torrente T., A. 2013. El Suelo - Propiedades Físicas. Ed. Universidad Surcolombiana. Neiva. 95 p.

13.Trujillo, O.E. 2011. Oferta edafica en un suelo de la Asociación Zarzal (Za), bosque seco tropical - Valle del Cauca, bajo pastoreo de bovinos. Tesis Maestría, Universidad Nacional de Colombia sede Palmira. Valle del Cauca. 112 p.

14.USDA, 2006. Claves para la Taxonomía de Suelos, Departamento de Agricultura de los Estados Unidos. Servicio de Conservación de Recursos Naturales, Décima Edición, 339 p.

15. Vásquez, J. 2009. Caracterización de la Variabilidad Espacial de las Propiedades Físicas y Químicas en los Suelos del Centro Experimental de la Universidad del Magdalena. Santa Marta. 120 p. 
